\title{
Hiper-Homocisteinemia e Doenças Vaso-Oclusivas
}

\author{
Sergio Paulo Bydlowski, Antonio Carlos Magnanelli, Dalton de Alencar F. Chamone
}

São Paulo, SP

O termo tiol ou sulfidril refere-se ao grupo SH. Os tióis biológicos são reativos e ubíquiitos, e têm sido extensivamente estudados em vários sistemas biológicos, principalmente os aminotióis homocisteína, cisteína e glutationa.

A homocisteína é um pequeno aminoácido sulfidrílico com um peso molecular de $135,1^{1}$, que ocupa uma posição regulatória central no metabolismo da metionina por determinar o destino do seu aminoácido precursor, a metionina, no ciclo metil, compreendendo reações de transmetilação; além disto, a homocisteína, via transulfuração, participa na biossíntese da cisteína ${ }^{2}$. As funções essenciais do ciclo, de modo geral, incluem o fornecimento de metionina para síntese protéica, a reciclagem do metiltetra-hidrofolato, o metabolismo da colina (via betaína), e a geração de precursores para reações de metilação (S-adenosilmetionina), formação de poliaminas ( $\mathrm{S}$-adenosilmetionina), e biossíntese de cisteína (via homocisteína) ${ }^{3-6}$. Deficiências enzimáticas hereditárias ou adquiridas, tanto na via de remetilação da homocisteína, a metionina ou na sua transulfuração, a cisteína, resultam em níveis elevados de homocisteína e na síndrome clínica da hiper-homocisteinemia ou homocistinúria $^{2-5}$. Deficiências de co-fatores também podem causar esta síndrome.

Há quase 30 anos, Kilmer McCully, um patologista do Massachussets General Hospital, propôs que níveis plasmáticos elevados de homocisteína estariam associados com o desenvolvimento de lesões vasculares, baseando sua conclusão nos achados de autópsia de uma criança de 7,5 anos de idade, que tinha um nível plasmático de homocisteína muito alto devido a um raro defeito na via metabólica da vitamina $B_{12}$. A autópsia das artérias dessa criança revelou o que, superficialmente, pareciam lesões ateroscleróticas rapidamente progressivas. A análise microscópica de artérias pequenas, médias e grandes, mostrou placas fibrosas e focais na íntima, que freqüentemente rompiam a membrana elástica interna. As lesões diferiam das lesões ateroscleróticas usuais, no sentido que continham muito pouco lípides e eram rodeadas por uma proliferação intensa do tecido conectivo perivascular. McCully só havia visto lesões similares, anteriormente, em indivíduos com homo-

Faculdade de Medicina da Universidade de São Paulo e Fundação Pró-Sangue Hemocentro de São Paulo

Correspondência: Sergio Paulo Bydlowski - Divisão de Pesquisa e Biologia Molecular - Av. Dr. Enéas C. Aguiar, 155 - $1^{\circ}$ - 05403-000 - São Paulo, SP

Recebido para publicação em 13/2/98

Aceito em 15/4/98 cistinúria devido a defeitos no gene da cistationina $\beta$ sintetase. Baseado nesta similaridade, concluiu que uma elevada concentração de homocisteína, homocistina ou um derivado da homocisteína, era o fator comum que conduzia à lesão arterial.

McCully trabalhou por 10 anos no Departamento de Patologia da Harvard Medical School, mas teve dificuldade em convencer seus colegas sobre sua teoria. A despeito de seus esforços em desenvolver um modelo animal para o estudo da homocisteína e doença vascular, não conseguiu causar entusiasmo em relação a suas idéias e foi demitido da Harvard em 1979.

Hoje em dia, apesar da relativa falta de informação sobre a fisiopatologia da doença vascular causada pela homocisteína, está claro que níveis plasmáticos elevados de homocisteína são um fator de risco independente para a doença arterial coronária, doença vascular periférica, doença cerebrovascular e trombose venosa. Antes de revisarmos os dados que embasam estas afirmações, abordaremos brevemente o metabolismo da homocisteína.

\section{Metabolismo da homocisteína}

A homocisteína é derivada da metionina proveniente da dieta e é um metabólico intermediário na via biossintética que converte a metionina em cisteína. A cisteína, portanto, não é um aminoácido essencial em humanos, uma vez que pode ser sintetizada a partir da metionina, via processo de transulfuração. A figura 1 mostra, esquematicamente, a via metabólica da homocisteína. A metionina dietética é convertida a S-adenosilmetionina (AdoMet) pela enzima metionina-adenosiltransferase (MAT). MAT forma uma ligação de enxofre de alta energia entre o carbono 5' da ribose do ATP e o enxofre da metionina. Uma pequena quantidade da AdoMet é utilizada para a síntese de poliaminas, e a maior parte doa seu grupo metil a outros compostos, incluindo creatina, DNA e RNA; deste modo, AdoMet é metabolizada em S-adenosil-homocisteína que, por sua vez, é hidrolisada à homocisteína e adenosina. Indivíduos homozigotos para defeitos no gene que expressa a forma hepática da MAT possuem níveis plasmáticos extremamente altos de metionina $(250$ a $1270 \mu \mathrm{moL} / \mathrm{L}$; normal: $<30 \mu \mathrm{moL} / \mathrm{L})$ e níveis plasmáticos normais de homocisteína, não apresentando doença cardiovascular ${ }^{7}$. Assim, níveis elevados de metionina não estão associados à doença vascular.

A homocisteína sintetizada, a partir da metionina, tem dois destinos possíveis: metabolização pela via da transulfuração, a cistationa, ou pela via de remetilação, a metionina ${ }^{8}$. O 


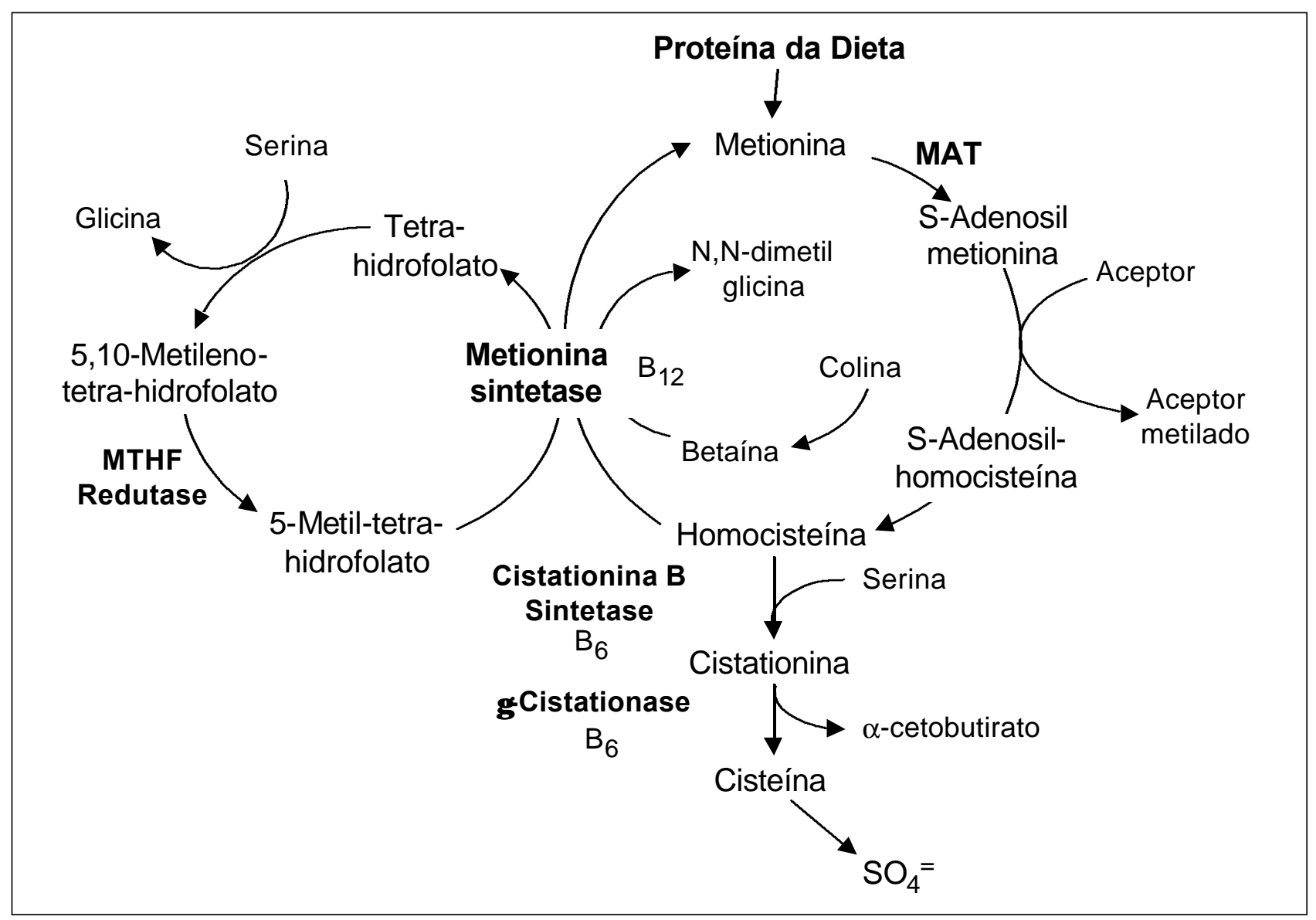

Fig. 1 - Esquema da via metabólica da homocisteína

requerimento de metionina para estas reações metabólicas é em grande parte proveniente da sua própria capacidade em se regenerar, a partir da homocisteína, prevenindo, portanto, sua depleção. A via de transulfuração, na qual a homocisteína é irreversivelmente convertida à cisteína, existe como uma exceção a esta regra.

Aproximadamente $50 \%$ da homocisteína é convertida, irreversivelmente, à cistationina, reação catalizada pela enzima cistationina $ß$-sintetase (CBS), dependente do fosfato de piridoxal (vitamina $\mathrm{B}_{6}$ ), como co-fator, pela chamada via de transulfuração. Nesta reação, a homocisteína sofre uma condensação com serina para formar um tioéter, a cistationina. A reação é alostérica e positivamente regulada pela AdoMet, o que serve para promover a depleção de um excesso de homocisteína, quando os níveis plasmáticos de metionina estão altos. A enzima seguinte na reação, $\gamma$ cistationase, hidrolisa a cistationina para gerar cisteína e $\alpha$ cetobutirato. Esta enzima, como a CBS, é dependente da vitamina $\mathrm{B}_{6}$.

A homocisteína remanescente, formada da metionina, é remetilada para regenerar metionina. Duas diferentes enzimas catalizam esta reação, mas a mais importante é a 5metiltetrahidrofolato-homocisteína metiltransferase, também chamada de metionina sintetase. Esta enzima é encontrada em todas as células e requer, como co-fator, a vitamina $\mathrm{B}_{12}$. A reação é acoplada à conversão do 5-metiltetra- hidrofolato (a forma circulante do folato reduzido) a tetrahidrofolato, que então entra nas células. A geração do 5metiltetra-hidrofolato por esta reação requer a redução do 5, 10-metilenotetra-hidrofolato a 5-metiltetra-hidrofolato, que é catalisado pela 5, 10-metilenotetra-hidrofolato redutase (MTHFR). MTHFR é inibida alostericamente pela AdoMet; assim, altos níveis de metionina previnem a formação de 5metiltetra-hidrofolato ${ }^{9} \mathrm{e}$, indiretamente, a síntese de metionina a partir da homocisteína.

Em seres humanos, a remetilação pode ser efetivada por vias alternativas. A betaína, que é derivada da colina da dieta, também pode atuar como um doador de metil na conversão da homocisteína em metionina. Esta reação é catalizada pela betaína-homocisteína metiltransferase, que é uma outra enzima dependente da vitamina $\mathrm{B}_{12}$, encontrada em quantidades significantes somente no fígado. $\mathrm{O}$ papel relativo desta via na regeneração da metionina em humanos não é conhecido.

A homocisteína participa ativamente em grande variedade de reações bioquímicas fora do ciclo metil e pode determinar a presença de tióis reduzidos. Participa em reações de radicais dissulfetos com proteínas ${ }^{10,11}$, assim como com outros tióis ${ }^{12}$ e sofre conversão reversível a tiolactona ${ }^{13}$. O equilíbrio entre tióis ligados a proteínas e tióis livres favorece os primeiros, o que representa, conseqüentemente, o principal pool de homocisteína in vivo ${ }^{10}$; a relação entre homo- 
cisteína e tiolactona depende do $\mathrm{pH}$ e da disponibilidade de vitamina $B_{12}{ }^{10,13}$. Vias alternativas no metabolismo da homocisteína incluem sua oxidação à homocistina e ácido homocistéico ${ }^{6,12}$.

A conversão da homocisteína em cistationina ou metionina mantém baixa a concentração intracelular de

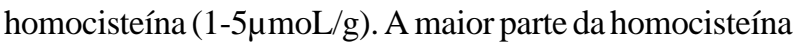
na célula apresenta-se no estado reduzido (homocisteína), ao invés de estar ligado por ligações dissulfetos para formar homodímero (homocistina) ou a outras proteínas para formar dissulfetos mixtos. O excesso intracelular de homocisteína é exportado da célula para o plasma.

Aproximadamente $70 \%$ da homocisteína que circula no plasma está ligada a proteínas, na maior parte à albumina. Em indivíduos normais, os níveis plasmáticos de homocisteína total variam entre 7 a $14 \mu \mathrm{moL} / \mathrm{L}$, com um nível médio de $11,6 \mu \mathrm{moL} / \mathrm{L}^{14}$. Porém, este valor depende da técnica utilizada para a determinação. Aproximadamente 20-30\% da homocisteína plasmática não está ligada à proteína e na sua maior parte circula como homocisteína ou dissulfetos mixtos homocisteína-cisteína. A quantidade de homocisteína ligada às proteínas plasmáticas é saturada em cerca de $100 \mu \mathrm{moL} / \mathrm{L}$ de homocisteína livre ${ }^{15}$. Assim, em indivíduos com níveis plasmáticos acima deste valor, a homocisteína livre pode representar uma porcentagem significante da homocisteína circulante total ${ }^{16}$.

A controvérsia persiste em relação à nomenclatura para descrever níveis "totais" de homocisteína. Mudd e Levy ${ }^{16}$ propuseram que quando a homocisteína total é medida (ligada e não ligada à proteína), deve-se utilizar o termo homocist(e)ína, desde, que assim ambas as formas são incluídas, as ligadas por dissulfeto e a sulfidril livre. Eles argumentaram que esta não é uma simples questão de semântica, pois que a concentração plasmática da forma sulfidril livre (reduzida) aumenta com o aumento dos níveis totais de homocisteína e pode ser muito importante na patogenia da formação da lesão vascular. Esta nomenclatura, embora tecnicamente correta, não tem sido utilizada de modo geral; de qualquer modo, homocist(e)ína é sinônimo de homocisteína total.

Estudos iniciais mediam somente a fração não ligada à proteína, ácido-solúvel, da homocisteína. Porém, desde que a taxa de homocisteína ligada/não ligada muda com o armazenamento, determinações fidedignas dos níveis plasmáticos usando estes métodos eram problemáticas. Os métodos atuais para medir a homocisteína plasmática total sujeitam as amostras de plasma a redução para liberar todas as ligações dissulfeto antes da determinação da quantidade total de homocisteína ${ }^{14}$. De qualquer modo, taxas fidedignas de níveis plasmáticos normais de homocisteína não têm sido estabelecidas e bons padrões de referência para calibrar ensaios em diferentes laboratórios não estão disponíveis, o que dificulta as comparações inter-laboratoriais.

Apesar disto, sabe-se que os níveis plasmáticos de homocisteína total variam conforme a idade e o sexo do indivíduo. Homens e mulheres pós-menopausa apresentam ní- veis maiores de homocisteína do que mulheres pré-menopausa, apesar disto não ser um achado universal ${ }^{14}$. Crianças tendem a apresentar níveis mais baixos ${ }^{15,17}$, sendo que os níveis tendem a crescer com a idade, enquanto que níveis de cistationina sintetase podem diminuir com a idade ${ }^{18}$. Estudos epidemiológicos demonstraram uma correlação negativa entre homocisteína e vitamina $B_{12}$ plasmática ${ }^{19} \mathrm{e}$ uma correlação positiva forte com a idade, sexo masculino, fumo, hipertensão arterial, níveis aumentados de colesterol e sedentarismo ${ }^{20}$. Assim, para a comparação plasmática dos níveis de homocisteína entre os grupos, os pacientes devem ter controles apropriados referentes à idade, sexo e estado estrogênico.

\section{Causas de hiper-homocisteinemia}

Hereditárias-O termohiper-homocisteinemia familiar foi introduzido na literatura por Genest e $\mathrm{col}^{21}$, em um trabalho que analisava dados de estudo de pais e parentes de pacientes com doença coronária prematura.

Hiper-homocisteinemia grave é usualmente resultado de deficiência homozigótica da cistationina- $\beta$-sintetase (CBS). O gene desta enzima é herdado de modo recessivo autossômico e demonstra marcada heterogeneidade genética. A incidência da deficiência homozigóticaé estimada em 1 em cada 335.000 nascimentos ${ }^{22}$. Pacientes homozigotos para a deficiência de CBS desenvolvem a clássica síndrome de homocistinúria, incluindo doença vascular prematura e trombose, retardo mental e anormalidades esqueléticas. $\mathrm{O}$ gene para CBS está localizado no cromossomo $21^{23}$. Pacientes com trissomia 21 possuem atividade enzimática de até $166 \%$ do normal; é interessante que estes pacientes raramente desenvolvem doença aterosclerótica ${ }^{24}$. Em anos recentes, verificou-se que a herança de somente um gene CBS funcional pode resultar também em hiper-homoscisteinemia, apesar do grau moderado. A incidência de deficiência heterozigótica de CBS é estimada entre 0,3 e 1,0\% da população geral.

Deficiências de enzimas da via da remetilação também podem resultar em hiper-homocisteinemia. Como já visto, a enzima metilenotetra-hidrofolato redutase (MTHFR) catalisa a conversão de 5,10-metilenotetra-hidrofolato a 5metiltetra-hidrofolato. Deficiência homozigótica desta enzima ocorre na população geral numa taxa de $1 / 10$ da deficiência de $\mathrm{CBS}^{25}$. Pacientes com deficiência homozigótica não apresentam atividade enzimática e desenvolvem hiperhomocistenemia e síndrome clínica consistindo de disfunção neurológica, retardo psico-motor, epilepsia e neuropatia periférica. Autópsias de pacientes homozigóticos revelaram uma alta incidência (70\%) de tromboses arteriais e venosas ${ }^{26}$. A deficiência heterozigótica resulta em cerca de $50 \%$ da atividade enzimática normal, o que aparentemente é adequado para a proteção contra deficiências neurológicas.

Kang e col descreveram um mutante termo-lábil do MTHFR que ocorre em pacientes neurologicamente normais ${ }^{27}$; de 16 pacientes que eram heterozigotos obrigatórios para deficiência severa de MTHFR, quatro tinham herdado 
um gene da variante termo-lábil. Esta enzima possui 50\% da atividade normal da MTHFR e pode resultar em elevação moderada dos níveis sangüíneos de MTHFR ${ }^{28}$. A forma termolábil da enzima(MTHFR-T) resulta da transição $677 \mathrm{C} \rightarrow \mathrm{T}$ no gene da MTHFR (Ala $677 \rightarrow$ Val) e compromete o sítio de ligação com o ácido fólico que, uma vez não ativo, dificulta a metabolização da homocisteína. Homozigotos para MTHFRT têm níveis de homocisteína 2,5 vezes superiores aos valores observados entre indivíduos normais ou heterozigotos. O diagnóstico da MTHFR-T é realizado pela PCR e posterior digestão do produto de amplificação do gene com a endonuclease Hinf I.

A prevalência da homozigose para MTHFR é comum na população geral, sendo detectada entre 5-15\% das populações caucasóides japonesas e do Oriente Médio, mas menos frequiente (1-2\%) entre populações africanas ${ }^{29,30}$. No Brasil foi verificado que apenas $4 \%$ de 296 indivíduos eram homozigotos para a variante termolábil desta enzima ${ }^{31}$, mas nenhuma correlação com os níveis plasmáticos de homocisteína foi estudada.

Adquiridas - Existem vários relatos em que a deficiência de cobalamina, folato ou piridoxina resulta em níveis sangüíneos elevados de homocisteína.

Em 1969, McKully descreveu uma criança com metabolismo anormal de cobalamina, com níveis séricos elevados de homocisteína e falecida com a idade de 7,5 semanas ${ }^{32}$. Brattström e col ${ }^{33,34}$ mediram os níveis séricos totais de homocisteína em 20 pacientes com deficiência assintomática $\mathrm{deB}_{12}, 21$ controles normais e 14 heterozigotos para deficiência de CBS. O grupo deficiente em $\mathrm{B}_{12}$ tinha níveis médios de homocisteína duas vezes maior que os controles, e maiores que os heterozigotos. Duas semanas após injeção intramuscular de $1 \mathrm{mg}$ de hidroxicobalamina, os níveis de homocisteinemia se normalizaram nos pacientes com deficiência de vitamina $B_{12}$. Stabler e col descreveram que 77 de 78 indivíduos com deficiência de $\mathrm{B}_{12}$ apresentavam níveis basais de homocisteína maiores que o normal ${ }^{35}$. Esses relatos sugerem que a elevação de homocisteína pode também ser usada como método altamente sensível para detectar pacientes com deficiência sub-clínica de $\mathrm{B}_{12}$, mesmo em certos casos nos quais os níveis séricos de cobalamina são normais ${ }^{36,37}$.

A deficiência de folato também está comumente associada à hiper-homocisteinemia, presumivelmente por atuação na via da remetilação. De 19 indivíduos deficientes em folato, 18 tinham valores de homocisteinemia maiores que o normal, na investigação de Stabler e $\operatorname{col}^{35}$. Kang e col encontraram níveis de homocisteína 4 vezes maiores em $84 \%$ de pacientes com níveis de folato subnormais ${ }^{38}$.

A deficiência de vitamina $\mathrm{B}_{6}$ tem sido relatada como um possível fator de risco para a aterosclerose no ser humano, apesar do mecanismo não estar esclarecido. Há relatos de pacientes com doença vascular arterial cerebral, carotídea e aorto-ilíaca que apresentam níveis baixos de fosfato de piridoxal ${ }^{39}$. Esta enzima é coenzima para CBS e, em estudos animais, a deficiência de vitamina $\mathrm{B}_{6}$ pode re- sultar em atividade diminuída de CBS. Ratos deficientes em vitamina $B_{6}$ desenvolveram níveis aumentados de homocisteína, corrigidos quando são tratados com vitamina $\mathrm{B}_{6}{ }^{40}$. Em estudo em humanos realizado há mais de 20 anos, 6 indivíduos foram alimentados com dietas deficientes em vitamina $B_{6}$ por 21 dias; a excreção urinária de homocisteína foi aumentada substancialmente após sobrecarga de metionina ${ }^{41}$. Entretanto, em um estudo mais recente da Universidade de Tufts, 11 pacientes idosos consumiam dietas deficientes em vitamina $\mathrm{B}_{6}$ por até 20 dias ${ }^{42}$. A deficiência de vitamina $\mathrm{B}_{6}$ foi documentada em todos os pacientes por excreção urinária aumentada de ácido xanturênico após sobrecarga de triptofano. Somente um paciente apresentou níveis basais elevados de homocisteína. Estes últimos resultados sugerem um papel secundário da deficiência de $\mathrm{B}_{6}$ na etiologia da hiperhomocisteinemia, mas mais estudos devem ser realizados.

Outras etiologias relacionadas à hiper-homocisteinemia também foram descritas: pacientes com insuficiência renal crônica ${ }^{43}$ ou aqueles pós-transplante renal ${ }^{44}$ apresentam níveis aumentados de homocisteinemia livre e ligada a proteínas. O mecanismo presumível da hiper-homocisteinemia, nestes casos, é a excreção renal diminuída de homocisteína, ou seu metabolismo diminuído. Outros fatores como deficiência de zinco, leucemia, psoríase e drogas anti-folato, também têm sido associados à hiper-homocisteinemia ${ }^{8}$. Entretanto, a significância clínica destas associações é desconhecida.

\section{Papel da homocisteína na doença vascular}

Atenção crescente tem sido dada à hiper-homocisteinemia como um fator etiológico-metabólico para a doença vascular prematura e trombose.

Como vimos anteriormente, esta associação foi inicialmente proposta por McCully, em $1969^{32}$. As lesões arteriais em pacientes que morrem de homocistinúria mostram hiperplasia proeminente da íntima, vacuolização, descamação endotelial e fibrose; trombos murais organizados comprometem a luz vascular já estreitada. McCully e Wilson propuseram, posteriormente, que elevações moderadas nos níveis de homocisteína poderiam ser causas importantes de aterosclerose $^{45}$.

Wilcken e Wilcken foram os primeiros investigadores que compararam níveis de homocisteína em pacientes com doença vascular aterosclerótica com controles ${ }^{46}$. Homens abaixo de 50 anos de idade com doença coronária documentada por angiografia tinham níveis de homocisteína-cisteína após carga oral de metionina maiores do que indivíduos com artérias coronárias normais. De fato, alguns indivíduos tinham, inclusive, níveis similares ao relatados para heterozigotos obrigatórios. Esse estudo sugeria que a elevação moderada de homocisteína era um possível risco adicional para doença coronária. Entretanto, Wilcken e col $^{47}$, subseqüentemente, não conseguiram reproduzir seus resultados. Os autores, revisando seus dados, verificaram que os pacientes com níveis elevados 
no primeiro estudo haviam recebido doses mais altas de metionina que os controles, e concluíram que o fornecimento de metionina por quilograma de peso resultava em falso-positivos em indivíduos com sobre-peso. Entretanto, outros pesquisadores não confirmaram correlação entre a dose total de metionina recebida e os níveis de homocisteína-cisteína ${ }^{48}$.

Num estudo similar, Murphy-Chutorian e col $^{49}$ estudaram 138 homens com idade entre 31-65 anos que foram a seu centro para cateterização cardíaca. Por análise de regressão multivariada, a homocisteinemia pós-carga de metionina foi definida como fator de risco para doença coronária, independentemente de idade, fumo, hipertensão, diabetes mellitus e colesterol sérico.

Olszewski e Szostak ${ }^{50}$ mediram os níveis totais de homocisteína usando o método da hidrólise ácida, em 26 homens, 2-3 meses após infarto agudo do miocárdio (IAM) e observaram uma elevação significante nos níveis de homocisteína em relação aos controles. Por outro lado, utilizando-se de metodologia similar, investigadores suecos não puderam observar diferenças ${ }^{51}$.

Foi relatado que a hiper-homocisteinemia ocorre em cerca de $30 \%$ dos pacientes com doença arterial oclusiva prematura ${ }^{52,53}$. Também a aterosclerose no transplante cardíaco foi estudada e não se observou correlação entre hiper-homocisteína e evidência angiográfica de aterosclerose coronária; porém a população estudada foi muito pequena ${ }^{54}$.

Dois estudos interessantes relataram valores de homocisteína total (a soma de homocistina, homocisteína, homocisteína-cisteína e homocisteína ligada à proteína) em pacientes com doença coronária, sem carga de metionina. No $1^{\circ}$ estudo ${ }^{55}$, o grupo de pacientes incluía 170 homens com idade <60 anos que apresentavam um grau de estenose de mais de $50 \%$. Os pacientes apresentavam aumento significante dos níveis de homocisteína total em relação aos controles $(13,66 \pm 6,44$ vs $10,93 \pm 4,92 \mu \mathrm{moL} / \mathrm{mL})$. A homocisteína total plasmática novamente foi considerada como um fator de risco independente para a doença coronária.

Num $2^{\circ}$ estudo, prospectivo, aproximadamente 15.000 médicos, sem história prévia de IAM ou acidente vascular cerebral forneceram sangue para análise no início do estudo ${ }^{56}$, sendo que 271 desenvolveram IAM e foram utilizados como grupo de pacientes. O nível de homocisteína total, sem carga de metionina, foi significantemente maior no grupo de pacientes.

Em resumo, a maior parte dos relatos publicados descrevem níveis elevados de homocisteína em pacientes com doença coronária. Em vários destes estudos, a hiperhomocisteinemia foi considerada como fator de risco independente, sendo que mesmo dentro de níveis considerados como normais, o risco de desenvolvimento de doença coronária aumenta com o aumento da homocisteína plasmática, independentemente de outros fatores.

Por outro lado, estudos avaliando a hiper-homocisteinemia moderada têm produzido resultados conflitantes.
Para verificar se heterozigotos obrigatórios apresentavam risco aumentado para IAM, Mudd e col ${ }^{57}$ enviaram questionários a pais e avós de crianças com homocistinúria, e concluíram que o risco relativo não era muito maior que os controles. Por outro lado, na Holanda, a prevalência de hiper-homocisteinemia moderada em pacientes jovens com doença arterial oclusiva é alta, sendo que a terapia com vitamina $\mathrm{B}_{6}+$ ácido fólico normalizou o metabolismo da homocisteína ${ }^{58}$.

A gravidade da aterosclerose e trombose em pacientes com doenças em membros inferiores também tem sido associada à hiper-homocisteinemia ${ }^{59}$. Segundo den Heijer e col $^{60}$, a hiper-homocisteinemia aumenta de 2-3 vezes o risco de trombose venosa. Franken e col ${ }^{61}$ descreveram que em pacientes com doença arteriosclerótica prematura é freqüentemente encontrado hiper-homocisteinemia, que pode ser reduzida ao normal em virtualmente todos os casos com tratamento com vitamina $\mathrm{B}_{6}$, ácido fólico e betaína. Fermo e col ${ }^{62}$ examinaram 107 pacientes com tromboembolismo venoso e 50 com doença oclusiva arterial, 3 meses após o evento e concluíram que a hiper-homocisteinemia moderada pode ter significância patogênica na doença oclusiva arterial e venosa, podendo ser incluída entre distúrbios hereditários.

O risco para o desenvolvimento da doença arterial ou venosa foi observado ser variável entre distintas populações, sendo que em muitos casos foi atribuída à deficiência de cistationina-beta-sintetase ${ }^{63}$. A trombose venosa e arterial foi também correlacionada com a homozigose para MTHFR. Engbersen e col ${ }^{64}$ verificaram que $28 \%$ dos pacientes com doença vascular prematura tinham metabolismo anormal da homocisteína, atribuída ao MTHFR termo-lábil. Rozen ${ }^{65}$ numa revisão, mostra que a análise molecular da MTHFR identificou algumas mutações raras associada ao fenótipo de sua deficiência severa e uma mutação comum foi encontrada em $35-40 \%$ dos alelos da população em geral, que foi proposto como fator de risco em algumas formas de doença cardiovascular (mutação $677 \mathrm{C} \rightarrow \mathrm{T}$ ).

A hiper-homocisteinemia pode também estar envolvida em outras patologias que levam à doença vascular. Por exemplo, parece estar associada com doença macrovascular em proporção significante em pacientes com diabetes não insulino-dependentes ${ }^{66}$.

A hiper-homocisteinemia também parece ser fator de risco independente para doença vascular em pacientes urêmicos ${ }^{67}$ e nos tratados com hemodiálise ${ }^{68}$. Hiperhomocisteinemia está presente em pacientes receptores de transplante renal, representando risco potencial para problemas cardiovasculares ${ }^{69}$. É interessante notar o risco aumentado de doença vascular nesses pacientes e especular se a hiper-homocisteína pode exercer um papel na doença vascular associada à insuficiência renal.

Os mecanismos pelos quais a homocisteína favorece o processo de aterosclerose e trombose não são conhecidos.

Os efeitos tóxicos diretos da homocisteína nas células endoteliais tem sido substanciadas por vários investigadores. A disfunção endotelial está presente em pacientes jovens com doença arterial periférica oclusiva e hiper- 
homocisteinemia $^{70}$. A hiper-homocisteinemia parece atuar como fator de risco independente para disfunção endotelial arterial ${ }^{70,71}$. Hiper-homocisteinemia experimental em primatas não humanos induz desendotelialização e espessamento da íntima ${ }^{73,74}$. Carga oral de metionina em humanos causa descamação de células endoteliais, evidenciado por endotelemia ${ }^{75}$. Células endoteliais de aorta bovina e umbilicais humanas expostas à homocisteína exibem liberação de creatinina de modo dose-dependente, captação de azul de tripano, descolamento celular e lise celular, todas manifestações de citotoxicidade ${ }^{76,77}$. Estudos mais detalhados dos mecanismos de citotoxicidade demonstraram que o processo é mediado por sulfidril, é criticamente dependente da disponibilidade de oxigênio, e é acelerado por metais ${ }^{2,77}$. A citoproteção pela catalase confirmou o mecanismo através de radicais livres e implicou o peróxido de hidrogênio como a espécie injuriosa nestes modelos experimentais ${ }^{77,78}$.

Os efeitos da homocisteína na função e sobrevida plaquetária são controversos. Dois anos após a descrição da homocistinúria como entidade clínica, Mc Donald e col ${ }^{79}$ mostraram que crianças com homocistinúria apresentavam aumento da adesão plaquetária que podia ser reproduzida pela adição de homocisteína ao sangue total normal, porém não observaram efeito na agregação plaquetária. Estudos posteriores, porém, não comprovaram estes efeitos. Estudos em relação à sobrevida plaquetária não foram menos ambíguos ${ }^{80}$.

Uma relação muito pouco entendida entre os metabolismos do colesterol e da homocisteína pode representar uma explicação adicional para a aterosclerose induzida pela homocisteína. Infusões de tiolactona de homocisteína aumentam os níveis de colesterol total e LDL-colesterol em coelhos, e a relação entre os níveis de colesterol e de homocisteína em humanos também foi postulada ${ }^{81}$. A inabilidade de pacientes com deficiência de cistationina sintetase em formar cisteína daria alguma luz ao mecanismo bioquímico desta relação obscura, segundo esses autores ${ }^{81}$ : os produtos finais dos metabolismos do colesterol e da cisteína são o ácido cólico e a taurina, excretados na bile como o ácido biliar conjugado, ácido taurocólico; portanto, a transulfuração impedida da homocisteína à cistina poderia levar à diminuição da eficiência do metabolismo de colesterol, sendo que a aterogenicidade promovida por níveis elevados de LDL seria maior em pacientes com homocistinúria. Além disto, a oxidação da LDL induzida pela homocisteína ${ }^{82,83}$ e a forte carga iônica de proteoglicanos expostos ${ }^{84}$ poderiam facilitar a captação vascular de LDL e levar a um padrão acelerado de aterosclerose; a tiolação da LDL pela tiolactona de homocisteína representaria um meio potencial adicional de modificação da LDL nesta doença, de significância não determinada. Por outro lado, segundo Blom e col ${ }^{85}$, o risco aumentado de aterosclerose na hiper-homocisteinemia não é devido ao aumento da peroxidação lipídica.

Boushey e col $^{86}$, numa revisão recente, sumarizaram um grande número de evidências convincentes, associando níveis elevados de homocisteína e doença vascular e, baseados nos dados da literatura, concluíram que o aumento de $5 \mu \mathrm{moL} / \mathrm{L}$ nos níveis de homocisteína total conferia o mesmo aumento de risco para doença vascular que um aumento de 20mg/dL nos níveis de colesterol total. Recentemente, Tonstad e col ${ }^{87}$ estudaram 155 crianças de ambos os sexos, entre 7 e 17 anos, com hipercolesterolemia familiar, e concluíram que níveis moderadamente elevados de homocisteinemia podem contribuir para o risco familiar de doença cardiovascular nesta doença e postulam a inclusão de recomendações dietéticas que afetem o metabolismo da homocisteína nestes pacientes.

A ativação de fatores de coagulação V e XII, induzida pela homocisteína ${ }^{88}$, pode contribuir para a predisposição trombótica da homocistinúria. A ativação do fator $\mathrm{V}$ é também indiretamente mediada e atribuída à indução, pela homocisteína, de uma protease ativadora do fator $\mathrm{V}^{88}$. Mais ainda, o mecanismo da proteína $C$ para a inativação dos fatores V e VIII é impedido em células endoteliais tratadas com homocisteína ${ }^{89}$. Isto é atribuído à possível ação da homocisteína como um inibidor competitivo da ligação da trombina com a trombomodulina. Dados mais recentes sugerem que a homocisteína reduz diretamente a expressão de trombomodulina em células endoteliais, com conseqüente inibição da atividade da proteína $\mathrm{C}^{90}$. Entretanto, as atividades dos fatores de coagulação não estão evidentemente aumentados em pacientes com homocistinúria ${ }^{73}$.

\section{Conclusão}

O fato de que vários defeitos genéticos diferentes, que causam aumento dos níveis plasmáticos de homocisteína, estarem associados à doença vascular e trombose, é a principal evidência de que a homocisteína per se é a responsável pela vasculopatia. Porém, relativamente pouco progresso tem sido feito para explicar a associação patogênica entre a homocisteína e a doença vascular. Dados convincentes desta associação ainda não estão disponíveis. Um problema com todos os estudos é que se pressupõe que a adição de várias formas de homocisteína na circulação in vivo, ou a células in vitro, tem o mesmo efeito que concentrações intracelulares aumentadas.

A hiper-homocisteinemia pode ser conseqüência, ao invés da causa, de um distúrbio metabólico responsável pela vasculopatia. Mais ainda, na maior parte dos estudos experimentais, as dosagens de homocisteína empregadas chegam a 100 vezes o valor encontrado no plasma de indivíduos com hiper-homocisteína. Porém, a despeito da falta de evidências diretas de que a homocisteinemia causa doença vascular, a consistência de achados de níveis plasmáticos moderadamente elevados de homocisteína em indivíduos com doença arterial ou venosa sugere uma relação causaefeito. Provar esta relação requer estudos prospectivos adicionais. O desenvolvimento de modelos animais adequados ou linhagem celulares com defeitos moleculares de várias enzimas da via metabólica da homocisteína, ainda não disponíveis, poderão tornar mais clara esta relação. 


\section{Referências}

1. Jakoby WB, Griffith OW - Sulfur and sulfur amino acids. Methods Enzymol 1994; 143: 366-76.

2. Stipanuk MH - Metabolism of sulfur-containing amino acids. Annu Rev Nutr 1986; 6: 179-209.

3. Grieco AJ - Homocystinuria: pathogenic mechanisms. Am J Med Sci 1997; 273: 120-32.

4. Finkelstein JD, Martin JD - Methione metabolism in mammals. Distribution of homocysteine between competing pathways. J Biol Chem 1984; 259: 9508-13.

5. Kraus JP - Cystathionine beta-synthase (human). Methods Enzymol 1987; 143: 388-94.

6. Finkelstein JD, Martin JJ, Harris BJ - Methionine metabolism in mammals. The methionine-sparing effect of cysteine. J Biol Chem 1988; 263: 11750-4.

7. Mudd SH, Levy HL, Skovby F - Disorders of transsulfuration. In: Scriver CR, Beaudet AL, Sly WS, Valle D, eds. - The Metabolic Basis of Inherited Disease. New York: McGraw Hill, 1995: 1887-912.

8. Ueland PM, Refsum H - Plasma homocysteine, a risk factor for vascular disease: Plasma levels in health, disease, and drug therapy. J Lab Clin Med 1989; 114 : 473-501.

9. Selhub J, Miller JW - The pathogenesis of homocysteinemia: interruption of the coordinate regulation by S-adenosylmethionine of the remethylation and transsulfuration of homocysteine. Am J Clin Nutr 1992; 55: 131-8.

10. Kang SS, Wong PWK, Cook HY, Norusis M, Messer JV - Protein-bound homocyst(e)ine. A possible risk factor for coronary artery disease. J Clin Invest 1986; 77: 1482-6

11. Lou MF, Poulsen LL, Ziegler DM - Cellular protein-mixed disulfides. Methods Enzymol 1987; 143: 124-9.

12. Farris MW, Reed DJ - High-perfomance liquid chromatography of thiols and disulfides: dinitrophenol derivatives. Methods Enzymol 1987; 143: 101-9.

13. Duerre JA, Miller CH - Preparation of L-homocysteine from L-homocysteine thiolactone. Anal Biochem 1966; 17: 310-5.

14. Ueland PM, Refsum H, Stabler SP, Malinow MR, Andersson A, Allen RH - Total homocysteine in plasma or serum: methods and clinical applications. Clin Chem 1993; 39: 1764-79

15. Ueland PM, Refsum H, Battström L - Plasma homocysteine and cardiovascular disease. In: Francis Jr RB, ed. - Atherosclerotic cardiovascular disease, hemostasis, and endothelial function. New York: Marcel Dekker, 1992: 183-236.

16. Mudd SH, Levy HL - Plasma homocyst(e)ine or homocysteine? N Engl J Med 1995; $333: 325$

17. Wilcken DEL, Gupta VJ - Cysteine - homocysteine mixed disulfide: differing plasma concentrations in normal men and women. Clin Sci 1979; 57: 211-5.

18. Gartler SM, Hornany SK, Motulsky AG - Effect of chronologic age on induction of cystathionine synthase, uropophyrinogen 1 synthase, and glucose-Gphosphate dehydrogenase activities in lymphocytes. Proc Natl Acad Sci USA 1981; 78: 1416-9

19. Dalery K, Lussier-Cacan S, Selhum J, Davingnon J, Latour Y, Genest J Homocysteine and coronary artery disease in French Canadian subjects: relation with vitamin B12, B6, pyridoxal phosphate and folate. Am J Cardiol 1995; 75: $1107-11$

20. Nygard O, Violset SE, Refsum H et al - Total plasma homocysteine and cardiovascular risk profile: the Hordaland Homocysteine Study. JAMA 1995; 274 : 1526-33.

21. Genest JJ, McNamara JR, Upson B et al - Prevalence of familial hyperhomocyst(e)inemia in men with premature coronary artery disease. Arterioscler Thromb 1991; 11: 1129-36.

22. Kang SS, Wong PWK, Malinow MR - Hyperhomocyst(e)inemia as a risk factor for occlusive vascular disease. Annu Rev Nutr 1992; 12: 279-98.

23. Skovby F, Krassikoff N, Francke U - Assignment of the gene for cystathionine-bsynthase to human chromosome 21 in somatic cell hybrids. Hum Genet 1984; 65: 291-4.

24. Brattström L, Englund E, Brun A - Does Down syndrome support homocysteine theory of arteriosclerosis? Lancet 1987; i: 391-2.

25. Kang SS, Wong PWK, Bock HGO, Horwitz A, Grix A - Intermediate hyperhomocysteinemia resulting from compound heterozygosity of methylenetetrahydrofolate reductase mutations. Am J Hum Genet 1991; 48: 546-51

26. Skovby F - Inborn errors of metabolism causing homocysteinemia and related vascular involvement. Haemostasis 1989; 19(suppl 1): 4-9.

27. Kang SS, Zhou J, Wong PWK, Kowalisyn J, Strokosch G - Intermediate homocysteinemia: a thermolabile variant of methylenetetrahydrofolate reductase. Am J Hum Genet 1988; 43: 414-21

28. Kang SS, Wong PWK, Susmano A, Sora J, Norusis M, Ruggie N - Thermolabile methylene-tetrahydrofolate reductase: an inherited risk factor for coronary artery disease. Am J Hum Genet 1991; 48: 536-45.
29. Motulsky AG - Nutritional ecogenetics: homocysteine-related arteriosclerotic vascular disease, neural tube defects, and folic acid. Am J Hum Genet 1996; 58: 17-20.

30. Ubbink JB, Vermaak WJH, Delport R, van der Merwe A, Becker PJ, Potgieter H Effective homocysteine metabolism may protect South African blacks against coronary heart disease. Am J Clin Nutr 1995; 62: 802-8.

31. Arruda VR, von Zuben PM, Chiaparini LC, Annichino-Bizzacchi JM, Costa FF The mutation Ala 677 @ Val in the methylene tetrahydrofolate reductase gene: a risk factor for arterial disease and venous thrombosis. Thromb Haemost 1997; 77: 818-21.

32. Mc Cully KS - Vascular pathology of homocysteinemia: implications for the pathogenesis of arteriosclerosis. Am J Pathol 1969; 56: 111-28

33. Brattström L, Israelsson B, Lindgärd F, Hultberg B - Higher total plasma homocysteine in vitamin B12 deficiency than in heterozygosity for homocystinuria due to cystathionine-b-synthase deficiency. Metabolism 1988; 37: 175-8.

34. Brattström L, Israelsson B, Hultberg B - Plasma homocysteine and methionine tolerance in early-onset vascular disease. Haemostasis 1989; 19(suppl 1): 35-44.

35. Stabler SP, Marcell PD, Podell ER, Allen RH, Savage DG, Lindenbaum J Elevation of total homocysteine in the serum of patients with cobalamin or folate deficiency detected by capillary gas chromatography-mass spectrometry. J Clin Invest 1988; 81: 466-74.

36. Lindenbaum J, Savage DG, Stabler SP, Allen RH - Diagnosis of cobalamin deficiency. II. Relative sensitivities of serum cobalamin, methylmalonic acid, and total homocysteine concentrations. Am J Hematol 1990; 34: 99-107.

37. Savage DG, Lindenbaum J, Stabler SP, Allen RH - Sensitivity of serum methylmalonic acid and total homocysteine determinations for diagnosing cobalamin and folate deficiencies. Am J Med 1994; 96: 239-46.

38. Kang SS, Wong PWK, Norusis M - Homocysteinemia due to folate deficiency. Metabolism 1987; 36: 458-62

39. Brattström L, Israelsson B, Norrving B et al - Impaired homocysteine metabolism in early-onset cerebral and peripheral occlusive arterial disease. Atherosclerosis 1990; 81: 51-60.

40. Smolin LA, Benevenga NJ - Accumulation of homocyst(e)ine in vitamin B6 deficiency: a model for the study of cystathionine-b-synthase deficiency. J Nutr 1982; 112: 1264-72.

41. Park KY, Linkswiler H - Effect of vitamin B6 depletion in adult man on the excretion of cystathionine and other methionine metabolites. J Nutr 1970; 100: $110-6$

42. Miller JW, Ribaya-Mercado JD, Russell RMet al -Effect of vitamin B6deficiency on fasting plasma homocysteine concentrations. Am J Clin Nutr 1992; 55: 1154-60.

43. Wilcken DEL, Gupta VJ - Sulphur containing amino acids in chronic renal failure with particular reference to homocysteine and cysteine-homocysteine mixed disulphide. Eur J Clin Invest 1979; 9: 301-7.

44. Wilcken DEL, Gupta VJ, Betts AK - Homocysteine in the plasma of renal transplant recipients: effects of cofactors for methionine metabolism. Clin Sci 1981; 61: 743-9.

45. McCully KS, Wilson RB - Homocysteine theory of arteriosclerosis. Atherosclerosis $1975 ; 22: 215-27$

46. Wilcken DEL, Wilcken B - The pathogenesis of coronary artery disease. A possible role for methionine metabolism. J Clin Invest 1976; 57: 1079-82.

47. Wilcken DEL, Reddy SG, Gupta VJ - Homocysteinemia, ischemic heart disease, and the carrier state for homocystinuria. Metabolism 1983; 32: 363-70.

48. Brattström LE, Hardebo JE, Hultberg BL - Moderate homocysteinemia - a possible risk factor for arteriosclerotic cerebrovascular disease. Stroke 1984; 15: 1012-16.

49. Murphy-Chutorian DR, Wexman MD, Grieco AJ - Methionine intolerance: a possible risk factor for coronary artery disease. J Am Coll Cardiol 1985; 6: 725-30.

50. Olszewski AJ, Szostak WB - Homocysteine content of plasma proteins in ischemic heart disease. Atherosclerosis 1988; 69: 109-13.

51. Andersson A, Isaksson A, Brattström L, Israelsson B, Hultberg B - Influence of hydrolysis on plasma homocysteine determination in healthy subjects and patients with myocardial infarction. Atherosclerosis 1991; 88: 143-51.

52. Kozich V, Kraus E, de Franchis R et al - Hyperhomocysteinemia in premature arterial disease: examination of cystathionine beta-synthase alleles at molecular level. Hum Mol Genet 1995; 4: 623-9.

53. Robinson K, Mayer EL, Miller DP et al - Hyperhomocysteinemia and low pyridoxal phosphate. Common and independent reversible risk factors for coronary artery disease. Circulation 1995; 92: 2825-30.

54. Ambrosi P, Barlatier A, Habib G et al - Hyperhomocysteinaemia in heart transplant recipients. Eur Heart J 1994; 15: 1191-5.

55. Genest JJ, Mc Namara JR, Salem DN, Wilson PWF, Schaefer EJ, Malinow MR Plasma homocyst(e)ine levels in men with premature coronary artery disease. J Am Coll Cardiol 1990; 16: 1114-9. 
56. Stampfer MJ, Malinow MR, Willett WC et al - A prospective study of plasma homocyst(e)ine and risk of myocardial infarction in US physicians. JAMA 1992; 268: 871-91.

57. Mudd SH, Havlik R, Levy HL, Mc Kusick VA, Feinleib MA - A study of cardiovascular risk in heterozygotes for homocystinuria. Am J Hum Genet 1981; 33: 883-93.

58. Van den Berg M, Franken DG, Boers GH et al - Combined vitamin B6 plus folic acid therapy in young patients with arteriosclerosis and hyperhomocysteinemia. J Vasc Surg 1994; 20: 933-40.

59. Van den Berg M, Stehouwer CDA, Bierdrager E, Rauwerda JA - Plasma homocysteine and severity of atherosclerosis in young patients with lower-limb atherosclerotic disease. Arterioscler Thromb Vasc Biol 1996; 16: 165-71.

60. den Heijer M, Blom HJ, Gerrits WB et al - Is hyperhomocysteinaemia a risk factor for recurrent venous thrombosis? Lancet 1995; 346: 127.

61. Franken DG, Doers GH, Blom HJ, Trijbels FJ, Kloppenborg PW - Treatment of mild hyperhomocysteinemia in vascular disease patients. Arterioscler Thromb 1994; 14: 465-70.

62. Fermo I, D’ Angelo VS, Paroni R, Mazzola G, Calori G, D'Angelo A - Prevalence of moderate hyperhomocysteinemia in patients with early-onset venous and arterial occlusive disease. Ann Intern Med 1995; 123: 747-53.

63. Clarke R, Daly L, Robinson K et al - Hyperhomocysteinemia: an independent risk factor for vascular disease. N Engl J Med 1991; 324: 1149-55.

64. Engbersen AM, Franken DG, Boers GH, Stevens EM, Trijbels FJ, Blom HJ Thermolabile 5,10 - methylenetetrahydrofolate reductase as a cause of mild hyperhomocysteinemia. Am J Hum Genet 1995; 56: 142-50.

65. Rozen R - Molecular genetic aspects of hyperhomocysteinemia and its relation to folic acid. Clin Invest Med 1996; 19: 171-8.

66. Munshi MN, Stone A, Fink L, Fonseca V - Hyperhomocysteinemia following a methionine load in patients with non-insulin-dependent diabetes mellitus and macrovascular disease. Metabolism 1996; 45: 133-5.

67. Chauveau P, Chadefaux B, Coude M et al - Hyperhomocysteinemia, a risk factor for atherosclerosis in chronic uremic patients. Kidney Int 1993; 41: S72-7.

68. Bachmann J, Tepel M, Raidt $\mathrm{H}$ et al - Hyperhomocysteinemia and the risk of vascular disease in hemodialysis patients. J Am Soc Nephrol 1995; 6: 121-5.

69. Massy ZA, Chadefaux-Vekemans B, Chevalier A et al - Hyperhomocysteinaemia: a significant risk factor for cardiovascular disease in renal transplant recipients. Nephrol Dial Transplant 1994; 9: 1103-8.

70. Van den Berg M, Boers GH, Franken DG et al - Hyperhomocysteinaemia and endothelial dysfunction in young patients with peripheral arterial occlusive disease. Eur J Clin Invest 1995; 25: 176-81.

71. Woo KS, Chook P, Lolin YI et al - Hyperhomocyst(e)inemia is a risk factor for arterial endothelial dysfunction in humans. Circulation 1997; 96: 2542-4.

72. Lentz SR, Sobey CG, Piegors DJ et al - Vascular dysfunction in monkeys with diet-induced hyperhomocyst(e)inemia. J Clin Invest 1996; 98: 24-9.

73. Harker LA, Slichter SJ, Scott CR, Ross R - Homocysteinemia, vascular injury and arterial thrombosis. N Engl J Med 1974; 291: 537-43.
74. Harker LA, Ross R, Slichter SJ, Scott CR - Homocysteine-induced atherosclerosis. The role of endothelial-cell injury and platelet response in its genesis. J Clin Invest 1976; 58: 731-41.

75. Mrhova O, Hladovec J, Urbanova D - Metabolic changes in the arterial wall and endothelial injury in experimental methioninaemia. Cor Vasa 1988; 30: 73-9.

76. De Groot PG, Willems C, Godfried H - Endothelial cell dysfunction in homocystinuria. Eur J Clin Invest 1983; 13: 405-10.

77. Wall RT, Harlan JM, Harker LA, Striker GE - Homocysteine-induced endothelial cell injury in vitro: a model for the study of vascular injury. Thromb Res 1980; 18: 113-21.

78. Starkebaum G, Harlan JM - Endothelial cell injury due to copper-catalyzed hydrogen peroxide generation from homocysteine. J Clin Invest 1986; 77: 1370-6.

79. McDonald L, Bray C, Field C, Love F, Davies B - Homocystinuria, thrombosis, and blood-platelets. Lancet 1964; i: 745-6.

80. Uhlemann ER, Ten Pas JH, Lucky AW, Schulman JD, Mudd SH, Shulamn NR Platelet survival and morphology in homocysteinuria due to cystathionine synthase deficiency. N Engl J Med 1976; 295: 1283-6.

81. Olszewski AJ, Szostak WB, Bialkowska M, Rudnicki S, Mc Cully KS Reduction of plasma lipid and homocysteine levels by pyridoxine, folate, cobalamin, choline, riboflavin, and troxeratin in atherosclerosis. Atherosclerosis 1989; 75: 1-6.

82. Heinecke JW, Rosen H, Suzuki LA, Chait A - The role of sulfur-containing amino acids in superoxide production and modification of low density lipoprotein by arterial smooth muscle cells. J Biol Chem 1987; 262: 10098-103.

83. Parthasarathy S - Oxidation of low-density lipoprotein by thiol compounds leads to its recognition by the acetyl LDL receptor. Biochim Biophys Acta 1987; 917: 337-40.

84. McCully KS - Importance of homocysteine-induced abnormalities of proteoglycan structure in arteriosclerosis. Am J Pathol 1979; 59: 181-93.

85. Blom HJ, Kleinveld HA, Boers GH et al - Lipid peroxidation and susceptibility of low-density lipoprotein to in vitro oxidation in hyperhomocysteinemia. Eur J Clin Invest 1995; 25: 149-54.

86. Boushey CJ, Beresford SAA, Omenn GS, Motulsky AG - A quantitative assessment of plasma homocysteine as a risk factor for vascular disease: probable benefits of increasing folic acid intakes. JAMA 1995; 274: 1049-57.

87. Tonstad S, Refsum H, Ueland PM - Association between plasma total homocysteine and parental history of cardiovascular disease in children with familial hypercholesterolemia. Circulation 1997; 96: 1803-8.

88. Rodgers GM, Kane WH - Activation of endogenous factor $\mathrm{V}$ by a homocysteineinduced vascular endothelial cell activator. J Clin Invest 1986; 77: 1909-16.

89. Rodgers GM, Conn MT - Homocysteine, an atherogenic stimulus, reduces protein C activation by arterial and venous endothelial cells. Blood 1990; 75: 895-901.

90. Lentz SR, Sadler JE - Inhibition of thrombomodulin surface expression and protein C activation by the thrombogenic homocysteine. J Clin Invest 1991; 88 : 1900-4. 